\section{Pulmonary edema on a chip}

\section{By Lauren Martz, Staff Writer}

Researchers at the Wyss Institute for Biologically Inspired Engineering at Harvard University have described the first disease model to emerge from the institute's organ-on-a-chip microfluidic device technology. ${ }^{1,2}$ The model of pulmonary edema could be better than culture models at predicting whether therapeutics will translate to humans.

The team already has used the lung-on-a-chip model in proofof-concept studies to test potential pulmonary edema therapeutic candidates including GlaxoSmithKline plc's GSK2193874, an inhibitor of transient receptor potential vanilloid 4 (TRPV4; VRL2), ${ }^{3}$ and wants to expand the use of the chip to model multiple lung diseases.

GSK2193874 was also proven effective in a mouse model of pulmonary edema, validating the results that were found in the chip model (see Box 1, “TRPV4 inhibition”).

The microfluidics system on a chip is about $2 \mathrm{~cm}$ long and mimics the alveolar-capillary interface and mechanical effects of breathing. The device is composed of two channels-a layer of human alveolar epithelial cells exposed to air and pulmonary microvascular endothelial cells exposed to flowing culture medium.

The channels are separated by a porous polymer-coated extracellular matrix. The channel interface is bordered by two chambers that can deform the polymer membrane upon application of a vacuum to mimic the mechanical effects of breathing.

Notably, the ability to simulate the mechanics of breathing is absent in $3 \mathrm{D}$ culture models of the lung.

Previous studies by the Boston team showed that the model can replicate the key physiological features of lung function, but it was unknown whether the device could model pulmonary diseases. ${ }^{2,4}$

Now, Donald Ingber and colleagues have generated a model that captures features of edema, provides insights into disease mechanisms and can help assess therapeutic candidates.

Ingber is a professor of vascular biology at Harvard Medical School and Boston Children's Hospital, professor of bioengineering at Harvard University and director of the Wyss Institute.

Pulmonary edema involves the abnormal accumulation of intravascular fluid in the alveolar air spaces and interstitial tissues of the lung. Most often it is the result of elevated intravascular pressure caused by heart disease or is a toxic side effect of some drugs, such as IL-2.

Indeed, Ingber and colleagues used IL-2 to develop their disease model. The team delivered a clinically relevant dose of the cytokine through the fluid microchannel and monitored fluid leak across the endothelium and into the alveolar air space.

IL-2 induced a fluid leak that continued for four days and decreased air volume compared with no treatment. The administration of both IL-2 and human blood plasma proteins caused fluid leak and fibrin clot formation that occur during the disease.

The application of mechanical strains to mimic breathing further compromised the barrier between the endothelium and alveolar epithelium and enhanced the leakage caused by IL-2. In contrast, mechanical strain in the absence of IL-2 did not alter barrier integrity.

These findings suggest breathing may exacerbate pulmonary edema and that artificial ventilation may be counterproductive in patients with pulmonary edema.

Finally, the Wyss group used the model to test potential therapeutics. Application of GSK2193874 or angiopoietin 1 (ANG1; ANGPT1), an antagonist of the edema-inducing ANG2 (ANGPT2), inhibited IL-2induced pulmonary edema.

Silence Therapeutics plc has Atu111, a small interfering RNA therapeutic targeting ANG2, in preclinical testing to treat acute lung injury.

The data were published in Science Translational Medicine. The authors included researchers from GSK and Seoul National University.

\section{Breathing easy}

The Boston team hopes to use the organ-on-a-chip technology to decrease the number of or replace many types of preclinical studies.

"Our next step is to explore what additional data might be required to convince pharmaceutical companies and the FDA that data produced by an on-chip model of human pulmonary edema could be used in place of results from an animal model of this condition to advance a drug toward testing in humans," Ingber told SciBX.

He said that unlike other in vitro models, the lung on a chip allows the study of organ-level functions. As for in vivo models, he said, "the chips should be faster and cheaper than animals."

Wolfgang Kübler, associate professor of surgery and physiology at the University of Toronto, was less bullish on the potential for taking animal studies out of the equation. "The model does not replicate animal experiments and the ability to study an entire organ or body," he said. "The litmus test still has to be in vivo, but this is certainly a good screening tool that may give a better indication of therapeutic success than simple cell cultures."
"The litmus test still has to be in vivo, but this is certainly a good screening tool that may give a better indication of therapeutic success than simple cell cultures."

\section{-Wolfgang Kübler University of Toronto}

be extendable to other lung conditions. For example, he said, the device could monitor the effects of aerosol-based toxins or aerosolized nanoparticles, aerosol-based drug delivery, smoke or chemical inhalation injury, radiation injury, fibrosis, pneumonias and metastasis. 


\section{Box 1. TRPV4 inhibition.}

While the Boston team tested Glaxo-

SmithKline plc's transient receptor potential vanilloid 4 (TRPV4; VRL2) inhibitor GSK2193874 in a lung-on-a chip model, the pharma reported on the discovery and subsequent animal studies of the edema therapeutic.

TRPV4 is known to mediate $\mathrm{Ca}^{2+}$ flux across the plasma membrane and promote vascular relaxation when activated by mechanical activities such as enhanced pressure. ${ }^{5,6}$

Previous studies, including work at the University of South Alabama, showed that knocking out Trpv4 in mice prevented increased vessel permeability and pressure-induced pulmonary edema. ${ }^{7}$ Now, the group from South Alabama has teamed up with GSK to develop a TRPV4 inhibitor.

In a paper published in Science Translational Medicine, ${ }^{3}$ Kevin Thorneloe and colleagues used small molecule screening and chemical optimization to develop GSK2193874. Thorneloe is a senior scientific investigator at GSK.

In isolated mouse and rat lungs, GSK2193874 reversed pulmonary edema. The therapeutic had no effect on the lungs at low pressure, suggesting it acts specifically against pressureinduced vessel leakage.

GSK2193874 also normalized pressure and decreased pulmonary edema compared with vehicle control in mouse models of chronic and acute venous pressure elevation. In the chronic model, GSK2193874 given one week after myocardial infarction-induced increases in pressure reversed pulmonary edema.

"GSK2193874 is still a discovery effort. Safety and toxicology studies are the next steps," said Thorneloe.

"TRPV4 is expressed in most cells in the body. This is a potential problem for inhibitors, and they may therefore be doing things that you don't want them to do, but the authors of the paper did a good job addressing some of the potential problems," said Wolfgang Kübler, associate professor of surgery and physiology at the University of Toronto. "For example, they showed that heart rate, blood pressure and kidney function were not affected by the inhibitors. They also showed that the effects of diuretics, which you would still want to give patients, were not altered."

He added, "This proves that if you block the TRPV4 channel, you can inhibit the further effects on fluid leakage. It also may support another pathological mechanism for TRPV4 that we have found. TRPV4 may also stimulate a cascade that inhibits the removal of fluid from the air spaces, so inhibiting the channel may both prevent leakage and increase fluid removal, but the team would need to confirm the relevance of this mechanism."

Thorneloe said GSK has filed for patents covering GSK2193874. - - LM
His team is working on models of small airways on a chip to test drugs for diseases including asthma, chronic obstructive pulmonary disease (COPD) and cystic fibrosis (CF). The small airway model is designed to include multilayered ciliated epithelium from small lung bronchioles lined by endothelium-future plans include integrating immune cells and airway smooth muscle cells into the model. The team is also in discussions with pharmaceutical and biotech companies to test other therapeutics using the organ-on-a-chip models.

"One limitation is the throughput. The technology is currently not suitable for high throughput screening. However, we do not see this limiting their application in the drug discovery and development process because we expect this technology to be used to produce high-quality, high-content predictive data. The data can be used to inform critical, high-value decisions such as selection or prioritization of a lead compound to move toward human testing," said Ingber.

Patent applications covering the chips, aerosol-based delivery, materials used in fabrication and different organ systems have been filed, and the IP is available for licensing.

Martz, L. SciBX 5(48); doi:10.1038/scibx.2012.1251

Published online Dec. 13, 2012

\section{REFERENCES}

1. Huh, D. et al. Sci. Transl. Med.; published online Nov. 11, 2012; doi:10.1126/scitranslmed.3004249
Contact: Donald E. Ingber, Harvard University, Boston, Mass. e-mail: don.ingber@wyss.harvard.edu

Contact: Geraldine A. Hamilton, same affiliation as above e-mail: geraldine.hamilton@wyss.harvard.edu

2. Huh, D. et al. Science 328, 1662-1668 (2010)

3. Thorneloe, K.S. et al. Sci. Transl. Med.; published online Nov. 7 , 2012; doi:10.1126/scitranslmed.3004276

Contact: Kevin S. Thorneloe, GlaxoSmithKline plc, King of Prussia, $\mathrm{Pa}$.

e-mail: kevin.s.thorneloe@gsk.com

4. Haas, M.J. SciBX 3(28); doi:10.1038/scibx.2010.847

5. Mendoza, S.A. et al. Am. J. Physiol. Heart Circ. Physiol. 298, H466H476 (2010)

6. Willette, R.N. et al. J. Pharmacol. Exp. Ther. 326, 443-452 (2008)

7. Jian, M.-Y. et al. Am. J. Respir. Cell Mol. Biol. 38, 386-392 (2008)

COMPANIES AND INSTITUTIONS MENTIONED

Boston Children's Hospital, Boston, Mass.

GlaxoSmithKline plc (LSE:GSK; NYSE:GSK), London, U.K. Harvard Medical School, Boston, Mass.

Harvard University, Boston, Mass.

Seoul National University, Seoul, South Korea

Silence Therapeutics plc (LSE:SLN), London, U.K.

University of Toronto, Toronto, Ontario, Canada

University of South Alabama, Mobile, Ala.

Wyss Institute for Biologically Inspired Engineering at Harvard University, Cambridge, Mass. 